\title{
AVALIAÇÃO DE CARACTERÍSTICAS BIOLÓGICAS DE TRICHOGRAMMA PRETIOSUM RILEY (HYMENOPTERA: TRICHOGRAMMATIDAE) CRIADO EM TRÊS HOSPEDEIROS
}

\section{H.X.L. Volpe, S.A. De Bortoli, R.T. Thuler, C.L.T.P. Viana, R.M. Goulart}

UniversidadeEstadual Paulista, Faculdade deCiências Agráriase Veterinárias, Departamento deFitossanidade, Via de Acesso Prof. Paulo Donato Castellane s/no, CEP 14884-900, Jaboticabal, SP, Brasil. E-mail: hxlvolpe@ig.com.br

\section{RESUMO}

\begin{abstract}
A importância do gênero Trichogramma no controle biológico tem intensificado sua utilização em muitos países, tornando cada vez mais relevante o conhecimento de seus hospedeiros. Dessa maneira, objetivou-se com este trabalho avaliar a especificidade de Trichogramma pretiosum em relação a três espécies hospedeiras habitualmente parasitadas, Anagasta kuehniella, Spodoptera frugiperda e Plutellaxylostella. O ensaio foi realizado no Laboratório de Biologia e Criação de Insetos da Faculdade de Ciências Agrárias e Veterinárias de Jaboticabal - UNESP. Foi realizado teste sem chance de escolha para o parasitóide em relação aos ovos daqueles hospedeiros. Verificou-se que para a primeira geração houve diferença significativa para o parâmetro biológico número de ovos parasitados, onde ovos de A. kuehniella foram os mais parasitados. Para a segunda geração, a porcentagem de emergência e o período de desenvolvimento ocorreu de forma análoga à da $1^{\mathrm{a}}$ geração nos 3 hospedeiros, não havendo diferenças estatísticas entre os eles. O maior número de ovos parasitados foi observado para os hospedeiros A. kuehniella e S. frugiperda, diferindo estatisticamente de P.xylostella. Pelos resultados conclui-se que a linhagem Tp- 8 de T. pretiosumtem melhor adaptação ao hospedeiro A. kuehniella apresentando condições para utilização em programas de controle biológico, tanto de S. frugiperda como de P. xylostella.
\end{abstract} PALAVRAS-CHAVE: Parasitóide de ovos, controle biológico, Trichogrammatidae, Plutellaxylostella,
Spodoptera frugiperda, Anagasta kuehniella.

\section{ABSTRACT}

EVALUATION OF BIOLOGICAL CHARACTERISTIC OF TRICHOGRAMMA PRETIOSUM RILEY (HYMENOPTERA: TRICHOGRAMMATIDAE) CREATE IN THREE HOST. Trichogramma genus are very important on biological control, since that its potential was discovered, its very useful worldwide as a option for pest control in different agroecosystems, making indispensable the knowledge of different hosts for these parasitoids. The aim of this work was to evaluate $T$. pretiosum specificity in relation to three host species. The research was conducted at Faculdade de Ciências Agrárias e Veterinárias de Jaboticabal (FCAV-UNESP), in the Laboratório de Biologia e Criação de Insetos. It was realized test of choice (no choice) in relation to Anagasta kuehniella, Spodoptera frugiperda and Plutella xylostella eggs handled under laboratory conditions. By the tests it was possible to verify differences for the number of parasitized eggs, being A. kuehniella preferred in the first and second generation, but no differences were founded for adults emergence. In the second generation, the higher number of parasitized eggs was observed for Anagasta kuehniella and Spodoptera frugiperda, differing of Plutella xylostella. By the results its possible to conclude that $T$. pretiosum $\mathrm{T}_{\mathrm{p}}-8$ strain had better adaptation to the host $A$. kuehniella and the strain of Trichogramma pretiosum was viable to be used in Biological Control programs, to control Spodoptera frugiperda and Plutella xylostella.

KEY WORDS: Eggs parasitoid, biological control, Trichogrammatidae, Plutella xylostella, Spodoptera frugiperda, Anagasta kuehniella.

\section{INTRODUÇÃO}

Parasitóides do gênero Trichogramma Westwood são utilizados em muitas culturas de interesse econômico para o controle de lepidópteros-praga em vários países, destacando-se a Rússia, China e México como os maiores produtores e usuários (LI, 1994).

Aproximadamente 18 espécies de Trichogramma são criadas massalmente (HASSANet al.,1998) em mais de 30 países, para liberações em 32 milhões de hecta- 
res (LI, 1994) no controle de pragas nas culturas do milho, cana-de-açúcar, arroz, soja, algodão, beterraba, hortaliças, maçã e em áreas de reflorestamento (HASsAn et al., 1998).

Os Trichogramatídeos constituem um importante grupo de inimigos naturais com potencial para o controle biológico por eliminarem a praga antes que qualquer dano seja causado à cultura (BLEICHER \& PARRA, 1989; Botelhoetal.,1995). Além disso, a adoção do controle biológico possibilita a diminuição do uso intensivo de inseticidas, o que tem contribuído para a implementação desse método de controle, juntamente com outros mais utilizados, como o químico (HAJI, 1992).

No Brasil, estudos com Trichogramma spp. começaram na década de 1940 para controle de Neoleucinodes elegantalis (Guenée) (Lepidoptera:Pyralidae) em tomate (Gomes, 1963). A partir daí o Brasil desenvolveu-se muito na área de controle biológico, motivado principalmente pelas informações geradas em trabalhos envolvendo diferentes espécies do parasitóide e também pela exigência do mercado por produtos livres de resíduos de agrotóxicos (THULER, 2006).

Graças à evolução brasileira na adoção do controle biológico de pragas são produzidos atualmente entre cinco e dez milhões de adultos por ano, liberados em cerca de 60.000 ha de milho e 1.200 ha de tomate e crucíferas, com perspectivas para aumento da utilização desse parasitóide (PARRA \&ZUCCHI, 2004).

Mundialmente são numerosos os trabalhos mostrando a gama de hospedeiros utilizados por diversas espécies de Trichogramma. Gómezet al.(1996) comprovaram que na Colômbia Trichogramma exiguum Pinto \& Platner (Hymenoptera: Trichogrammatidae) é a única espécie que parasita ovos de Diatraea spp. no campo. PARRA \& ZuCCHI (1997) afirmaram que na América do Sul quase a metade das espécies de Trichogramma estão associadas a um único hospedeiro. Na Argentina, Trichogramma fuentesi Torre (Hymenoptera: Trichogrammatidae) foi criado em laboratório e liberado, não havendo, no entanto, hospedeiros para a espécie em questão, em condições de campo. No Peru, essa espécie foi encontrada parasitando apenas ovos de Diatraea saccharalis (Fabricius) (Lepidoptera: Pyralidae). Também não foi encontrado hospedeiro para Trichogramma atropos Pinto (Hymenoptera: Trichogrammatidae) em condições de campo, na Venezuela.

Diante dessas afirmações torna-se imprescindível o desenvolvimento de trabalhos que demonstrem a especificidade hospedeira de linhagens de Trichogramma coletadas em campo, quando criados em hospedeiros naturais e alternativos. Assim, esse trabalho teve como objetivo avaliar algumas características biológicas de T. pretiosum, quando criado em ovos de A. kuehniella, P. xylostella e S. frugiperda.

\section{MATERIAL E MÉTODOS}

O ensaio foi realizado no Laboratório de Biologia e Criação de Insetos (LBCI) da Faculdade de Ciências Agrárias e Veterinárias de Jaboticabal - UNESP, sob temperatura de $25 \pm 1^{\circ} \mathrm{C}$, umidaderelativa de $70 \pm 10 \%$ e fotofase de $12 \mathrm{~h}$.

Para elaboração do experimento foram utilizados indivíduos do parasitóide de ovosT. pretiosum, linhagem Tp-8 da criação de Trichogramma do Laboratório de Entomologia do Centro de Ciências Agrárias da Universidade Federal do Espírito Santo (LE-CCA/ UFES). Como hospedeiros foram utilizados ovos da traça-das-farinhas, A. kuehniella (hospedeiro alternativo), adquiridos semanalmente junto à empresa BUG Agentes de Controle Biológico (Piracicaba, SP); ovos da lagarta-do-cartucho do milho, $S$. frugiperda (hospedeiro natural), obtidos no Laboratório de Ecologia Aplicada do Departamento de Fitossanidade da FCAV/UNESP e ovos da traça-das-crucíferas, $P$. xylostella (hospedeiro natural), procedente da criação estoque do LBCI.

A criação de T. pretiosum foi realizada em ovos de A. kuehniella colados com goma arábica (35\%) em cartelas de cartolina azul celeste e oferecidos ao parasitóide, que foram mantidos em tubos de vidro de fundo chato ( $8 \mathrm{~cm}$ de altura $\times 2 \mathrm{~cm}$ de diâmetro) fechados com filme plástico e acondicionados em BOD, à temperatura de $25 \pm 1^{\circ} \mathrm{C}$, umidade relativa de $70 \pm 10 \%$ e fotofase de $12 \mathrm{~h}$. Após $24 \mathrm{~h}$ de exposição ao parasitóide, essas cartelas foram separadas e colocadas em novos tubos de vidro até a emergência da geração seguinte, completando um novo ciclo.

No experimento, os ovos dos três hospedeiros foram expostos à ovoposição de T. pretiosum, sendo utilizados 30 ovos de P. xylostella, 30 de S. frugiperda e aproximadamente 100 ovos de A. kuheniella, que foram colados em cartelas de cartolina azul celeste $(0,4 \times 2,0 \mathrm{~cm})$. Apenas para A. kuheniella os ovos não foram contados, sendo então colados com goma arábica $35 \%$ numa área de $0,2 \mathrm{~cm}^{2}$ que, por amostragem, determinou-se ter aproximadamente 100 ovos.

As cartelas foram colocadas em tubos de centrifugação Eppendorf ${ }^{\circledR}$ de 2,0 mL contendo mel para alimentação do parasitóide e oferecidas a uma fêmea que foi individualizada da criação, compondo os seguintes tratamentos com 30 repetições:T. pretiosum parasitando A. kuehniella (testemunha), T. pretiosum parasitando P. xylostella e T. pretiosum parasitando S. frugiperda.

Foram avaliados o número de ovos parasitados, a porcentagem deemergência, o período de desenvolvimento e a longevidade de T. pretiosum nos 3 hospedeiros sendo os dados obtidos submetidos à analise de variância (testeF) e as médias confrontadas pelo teste de Tukey a $5 \%$ de probabilidade. 


\section{RESULTADOS E DISCUSSÃO}

Pela comparação das médias (Tukey, $p=0,05$ ) é possível notar a influência dos hospedeiros em relação ao número de ovos parasitados por T. pretiosum. O parasitismo em A. kuehiella foi o maior (15,6 ovos) fêmea), diferindo estatisticamente dos demais hospedeiros analisados (Tabela 1). Beserra \& PARra (2004) tiveram resposta contrária para número de ovos parasitados por fêmea de T. pretiosum, sendo maior o parasitismo em ovos deS.frugiperda (17) do que em $A$. kuehniella (7 ovos).

O número de ovos de A. kuehniella parasitados por T. pretiosumindica um provável condicionamento pré-imaginal do parasitóide, adquirido durante o desenvolvimento larval, o que, segundo COBERT (1985), pode ocorrer quando um parasitóide é criado por várias gerações no hospedeiro alternativo. Essa criação pode afetar a preferência pelo hospedeiro natural e alterar a sua eficiência no combate à praga alvo.

WÜHRER \& HASSAN (1993) não observaram correlação entre a freqüência de contatos do parasitóide com o ovo e parasitismo e detectaram uma diminuição na preferência de T. pretiosum por ovos de P. xylostella após sua criação em ovos de $S$. cerealella, reforçando a hipótese de condicionamento pré-imaginal.

Porcentagem de emergência, longevidade e período de desenvolvimento não sofreram interferência em função dos hospedeiros testados na geração $F_{1}$ (Tabela 1).

Resultados semelhantes para porcentagem de emergência de $T$. pretiosum foram encontrados por PRATISSOlietal. (2004) em ovos de A. kuhniella, eBESERRA \&PARra (2004) em ovos de S. frugiperda, sendo de $93 \%$ e $93,8 \%$, respectivamente. PEREIRA et al. (2004) encontraram porcentagem de emergência inferior para a mesma espécie de parasitóide, no entanto, em ovos de P. xylostella $(84,8 \%)$.

O período de desenvolvimento foi exatamente o mesmo para as três espécies hospedeiras estudadas (11 dias) (Tabela 1). Beserra \& PARRA (2004) observa- ram valor diferente na duração do ciclo ovo-adulto de T. pretiosum em ovos de $S$. frugiperda (9,6 dias), enquanto Pratissoliet al. (2000) verificaram um período de 10 dias para T. pretiosum parasitando ovos de $A$. kuehniella.

O aceleramento ou o retardamento do período de desenvolvimento do parasitóide depende da qualidade do hospedeiro, sendo que o conteúdo de ovos requerido por cada espécie e/ou linhagem desse parasitóide dependente das características genéticas, fenológicas e fisiológicas do hospedeiro em questão (LEwis \& MARTIN, 1990; GOMES, 1997; VINSON, 1997). Desta forma, a proximidade dos valores encontrados nesta pesquisa com resultados de outros trabalhos evidenciam que $P$. xylostella, $S$. frugiperda e A. kuehniella são hospedeiros adequados para o parasitóide.

PRATISSOli \& PARRA (2000) afirmaram que a variação na duração do ciclo (ovo-adulto) de espécies de Trichogramma, em determinada faixa de temperatura, registrado por diferentes autores, depende da adaptação da espécie ou linhagem do parasitóide ao hospedeiro utilizado.

A maior longevidade foi proporcionada pelo hospedeiro $P$. xylostella (7,6 dias), apesar deste valor não diferir dos encontrados para $S$. frugiperda (6,5 dias) e A. kuehniella (5,7 dias) (Tabela 1).

LEWis et al.(1976) encontraram maior longevidade em ovos deA. kuehniella do que em ovos deS. cerealella para Trichogramma sp. BERTI\& MARCANO (1993) obtiveram longevidade de $T$. pretiosum em ovos de $S$. cerealella similar à encontrada nesse estudo para $A$. kuehniella e BAI et al. (1995) mostraram que para Trichogramma brassicae Bezdenko (Hymenoptera: Trichogrammatidae), T. minutum e Trichogramma sibiricun Sorokina(Hymenoptera: Trichogrammatidae) ocorre variação na longevidade entre diferentes espécies (de 8,6 a 9,2 dias, de 10,2 a 11,7 e de 8,3 a 12,4, respectivamente), em ovos de A. kuehniella. Os resultados obtidos por diferentes autores mostraram que a longevidade pode variar em função do hospedeiro e da espécie de Trichogramma criada.

Tabela 1 - Número de ovos parasitados por fêmea, porcentagem de emergência, longevidade e período de desenvolvimento $( \pm \mathrm{EP})$ da geração $\mathrm{F}_{1}$ de Trichogramma pretiosum em ovos de três hospedeiros.

\begin{tabular}{|c|c|c|c|c|c|c|c|c|}
\hline Hospedeiros & $\mathrm{n}$ & $\begin{array}{l}\mathrm{N}^{\mathrm{o}} \text { de ovos } \\
\text { parasitados }\end{array}$ & $\mathrm{n}$ & Emergência (\%) & $\mathrm{n}$ & Longevidade (dias) & $\mathrm{n}$ & Período (dias) \\
\hline Anagasta kuehniella & 30 & $15,6 \pm 1,40 \mathrm{a}$ & 30 & $100 \pm 1,40 a$ & 30 & $5,7 \pm 0,53 \mathrm{a}$ & 30 & $11 \pm 0,00 \mathrm{a}$ \\
\hline Plutella xylostella & 28 & $10,8 \pm 1,02 b$ & 28 & $99,8 \pm 0,18$ a & 30 & $7,6 \pm 0,58 \mathrm{a}$ & 28 & $11 \pm 0,00 \mathrm{a}$ \\
\hline Spodoptera frugiperda & 30 & $11,9 \pm 0,75 b$ & 30 & $100 \pm 0,00 \mathrm{a}$ & 30 & $6,5 \pm 0,59 \mathrm{a}$ & 30 & $11 \pm 0,00 \mathrm{a}$ \\
\hline $\mathrm{CV}(\%)$ & & 45,24 & & 0,56 & & 46,91 & & 0,00 \\
\hline
\end{tabular}

Médias seguidas de mesma letra na coluna não diferem entre si pelo teste de Tukey $(p=0,05)$. 
Tabela 2 - Número de ovos parasitados por fêmea, porcentagem de emergência e período de desenvolvimento ( \pm EP) da geração $\mathrm{F}_{2}$ de Trichogramma pretiosum em ovos de 3 hospedeiros.

\begin{tabular}{lcccccc}
\hline Hospedeiros & $\mathrm{n}$ & $\begin{array}{l}\mathrm{N}^{\circ} \text { de ovos } \\
\text { parasitados }\end{array}$ & $\mathrm{n}$ & Emergência (\%) & $\mathrm{n}$ & Período(dias) \\
\hline Anagasta kuehniella & 27 & $14,8 \pm 1,24 \mathrm{a}$ & 27 & $100 \pm 0,00 \mathrm{a}$ & 27 & $10 \pm 0,00 \mathrm{a}$ \\
Plutella xylostella & 26 & $8,1 \pm 1,17 \mathrm{~b}$ & 26 & $99,6 \pm 0,30 \mathrm{a}$ & 26 & $11 \pm 0,00 \mathrm{a}$ \\
Spodoptera frugiperda & 25 & $18,6 \pm 1,81 \mathrm{a}$ & 25 & $100 \pm 0,00 \mathrm{a}$ & 25 & $10 \pm 0,00 \mathrm{a}$ \\
\hline CV $(\%)$ & \multicolumn{7}{c}{46,62} & 0,94 & - \\
\hline
\end{tabular}

Médias seguidas de mesma letra na coluna não diferem entre si pelo teste de Tukey $(p=0,05)$.

$\mathrm{Na}$ geração $\mathrm{F}_{2}$ o maior número de ovos parasitados foi observado para os hospedeiros $A$. kuehniella e $S$. frugiperda diferindo estatisticamente de P. xylostella; S. frugiperda apresentou maior valor absoluto (18,6 ovos parasitados) (Tabela 2). Esses resultados revelam uma tendência ao parasitismo em ovos de $S$. frugiperda após a criação do parasitóide nesse hospedeiro por uma geração. Segundo WAjnberg et al. (1989), Schmidt (1991) e PAK et al. (1986) a aceitação pelo hospedeiro não depende somente de herança genética, mas também de características herdadas ao longo de gerações. Esse fato poderia explicar o maior parasitismo em $A$. kuehniella na geração $\mathrm{F}_{1}$ (pois a criação foi mantida em A. kuehniella) e a tendência de aumentar o parasitismo em ovos de $S$. frugiperda na geração $\mathrm{F}_{2^{\prime}}$ após a manutenção do parasitóide nesse hospedeiro na $1^{\mathrm{a}}$ geração. Tal situação não ocorre para $P$. xylostella.

A porcentagem de emergência da geração $\mathrm{F}_{2}$ ocorreu de forma análoga à da primeira geração, nos três hospedeiros, não havendo diferenças estatísticas entre os mesmos (Tabela 2).

O maior período de desenvolvimento na $2^{\mathrm{a}}$ geração foi observado paraP. xylostella (11 dias), enquanto para S. frugiperda eA. kuehniella o período foi igual (10 dias), apesar dos tratamentos serem estatisticamente iguais (Tabela 2). As pequenas alterações no período de desenvolvimento para diferentes espécies de Trichogramma parasitando vários hospedeiros são comumente relatadas na literatura.

Os resultados de porcentagem de emergência e período de desenvolvimento, que não diferiram entre os hospedeiros, sugerem a adaptação do parasitóide aos mesmos.

\section{CONCLUSÕES}

§ A linhagem Tp-8 de T. pretiosum tem melhor adaptação ao hospedeiro A. kuehniella;

$\S$ A linhagem de T. pretiosum apresenta condições para utilização em programas de Controle Biológico, tanto de $S$. frugiperda como de P. xylostella.

\section{AGRADECIMENTOS}

Ao APECOLAB/FCAV-UNESP, Jaboticabal-SP, na pessoa do Prof. Dr. Odair Aparecido Fernandes, pelo fornecimento dos ovos deS. frugiperda utilizados no experimento.

\section{REFERÊNCIAS}

BaI, B.; Çobanoglu, S.; Smith, S.M. Assessment of Trichogramma species for biological control of forest lepidopteran defoliators. Entomologia Experimentaliset Applicata, v.75, p.135-145, 1995.

BERTI, J. \& Marcano, R. Effect of different food substances on the reproduction and lifespan of the female of Trichogramma pretiosum Riley (Hymenoptera: Trichogrammatidae). Boletin de Entomologia Venezolana, v.8, n.2, p.105-110, 1993.

BESERRA, E.B. \& PARRA, J.R.P. Biologia e parasitismo de Trichogramma atopovirilia Oatman \& Plantner e Trichogramma pretiosum Riley (Hymenoptera: Trichogrammatidae) em ovos de Spodopterafrugiperda (J. E. Smith) (Lepidoptera: Noctuidae). RevistaBrasileira de Entomologia, v.48, n.1, p.119-126, 2004.

BLEICHER,E.\&P ARRA,J.R.P.Espécies de Trichogrammaparasitóides de Alabamaargillacea. Biologia detrês populações. Pesquisa Agropecuária Brasileira, v.24, p.929-940, 1989.

Botelho, P.S.M.; Parra, J.R.P.; MagrinI, E.A.; Haddad, M.L.; ReSENDE, L.C.L. Parasitismo de ovos de Diatraeasaccharalis (Fabr., 1794) (Lep.: Pyralidae) por Trichogramma galloi Zucchi, 1988 (Hym.: Trichogrammatidae) em duas variedades de cana-de-açúcar conduzidas em dois espaçamentos de plantio. Revista Brasileirade Entomologia, v.39, p.591-595, 1995.

COBERT, S.A. Insect chemosensory responses: a chemical legacy hypothesis. Ecological Entomology, v.10, p.143153, 1985.

GOMES, J.G. Histórico do combate biológico no Brasil. In: SIMPÓSIO BRASILEIRO SOBRE COMBATE BIOLÓGICO, 1., 1963, Rio de Janeiro Boletim do Instituto Experimental do Estado do Rio, v.21, p.89-97, 1963.

GOMEs, S.M. Comparação de três hospedeiros alternativos para criação e produção massal de Trichogramma pretiosum e $T$. galloi. 1997. 106 f. Dissertação (Mestrado em Entomologia)-EscolaSuperior de Agricultura "Luizde Queiroz",Universidade deSão Paulo, Piracicaba.1997. 
Gómez, L.A.; DíAz, M.A.E.; Lastra, B.L.A. Reconocimiento de las especies de Trichogramma associadas con la caña de azúcar en Colombia. Revista Colombiana de Entomologia, v.22, n.1, p.1-5, 1996.

HAJI, F.N.P. Manejo de pragas do tomateiro no submédio São Francisco. In: Fernandes, O.A.; Correia, A. do C.B.; De Bortoli, S.A. Manejo integrado de pragas e nematóides. Jaboticabal: FUNEP, 1992. p.341-350.

Hassan,S.A.;H afes, B.O.; Degrande, P.E.;Herai, K. The sideeffects of pesticides on the egg parasitoid Trichogramma cacoeciae Marchal (Hym., Trichogrammatidae), acute dose-response and persistence tests. Journal of Applied Entomology, v.122, n.9-10, p.569-573, 1998.

Lewis, W.J.; Gross JR., H.R.; Perkins, W.D.; Knipling, E.F.; VOEGELÉ,J.Production and performance of Trichogramma reared on eggs of Heliothis zea and other hosts. Environmental Entomology, v.5, n.3, p.449-452, 1976.

Lewis, W.J. \& MARTIN, W.R. Semiochernicals for use with parasitoids: status and future. Journal ofChemical Ecology, v.16, p.3067-3089, 1990.

LI, L.Y. Wordwide use of Trichogramma for biological control on differents crops: a survey. In:WANJBERG,E.\&HASSAN, S.A. (Eds.). Biological control with eggs parasitoids. Wallingford: CAB International,1994. p.37-53.

PAK, A.G.; BuIs, H.C.E.M.; HeCK, I.C.C.; Hermans, M.L.G. Behavioral variations among strains of Trichogramma spp.: host-age selection. Entomologia Experimentalis et Applicata, v.40, n.3, p.247-258, 1986.

PARRA, J.R.P. \& Zucchi, R.A. (Eds.). Trichogramma e o controle biológico aplicado. Piracicaba: FEALQ, 1997.324p.

PARRA, J.R.P. \& ZuCCHI, R.A. Trichogramma no Brasil: viabilidade de uso após vinte anos de pesquisa. Neotropical Entomology, v.33, n.3, p.271-282, 2004.

Pereira, F.F.; B arros, R.; Pratissoli, D.; P arra, J.R.P. Biologia e exigências térmicas de Trichogramma pretiosumRiley e T. exiguum Pinto e Platner (Hymenoptera: Trichogrammatidae) criados em ovos de Plutella xylostella (L.) (Lepidoptera: Plutellidae). Neotropical Entomology, v.33, n.2, p.231-235, 2004.
Pratissoli, D.; Holtz, A.M.; Gonçalves, J.R.; Zanúncio, J.C. Influência do substratoalimentar dohospedeiroalternativo Anagasta kuehniella (Zeller, 1879), sobre Trichogramma pretiosum Riley, 1879. Ciência e Agrotecnologia, v.24, n.2, p.373-378, 2000.

Pratissoli, D. \& ParRa, J.R.P.Desenvolvimentoeexigências térmicas de Trichogramma pretiosum, criados em duas traças do tomateiro. Pesquisa Agropecuária Brasileira, v.35, p.1281-1288, 2000.

Pratissoli, D.; Holtz, A.M.; Gonçalves, J.R.; Oliveira, R.C.; VIANNA, U.R. Características biológicas de linhagens de Trichogramma pretiosum, criados em ovos deSitotroga cerealella e Anagasta kuehniella. Horticultura Brasileira, v.22, n.3, p.562-565, 2004.

SchmidT, J.M. The role of physical factors in tritrophic interactions. Redia, v.124, p.43-87, 1991.

Thuler, R.T. Plutella xylostella (L.) (Lepidoptera: Plutellidae) táticas para o manejo integrado em brássicas. 2006. 80f. Tese (Doutoradoem Agronomia - Entomologia Agrícola) - Faculdade de Ciências Agrárias e Veterinárias, Universidade Estadual Paulista, Jaboticabal, 2006.

VINSON, S.B. Comportamento de seleção hospedeira de parasitóide de ovos com ênfase na família Trichogrammatidae. In: PARRA, J.R.P. \& ZuCCHI, R.A. (Eds.). Trichogramma e o controle biológico aplicado. Piracicaba: FEALQ, 1997.324p.

WajnberG, E.; Pizzol, J.; Babault, M. Genetic variation in progeny allocation in Trichogramma maidis. Entomologia Experimentaliset Applicata, v.53, p.177-187, 1989.

WÜHRER, B.G. \& HASSAN, S.A. Selection of effective species / strains of Trichogramma (Hym.; Trichogrammatidae) to control the diamondback moth Plutella xylostella $\mathrm{L}$. (Lep., Plutellidae). Journal of Applied Entomology, v.116, p.80-89, 1993.

Recebido em 2/8/06

Aceito $\mathrm{rm} 4 / 9 / 06$ 